\title{
On Feedback Requirements for CoMP Joint Transmission in the Quasi-Static User Regime
}

\author{
Lars Thiele, Martin Kurras, Michael Olbrich and Kai Börner \\ Fraunhofer Heinrich Hertz Institute \\ Einsteinufer 37, 10587 Berlin, Germany \\ lars.thiele@hhi.fraunhofer.de
}

\begin{abstract}
In this work we focus on the downlink direction of cellular multi-user MIMO systems based on OFDM waveforms. Our target system is operated in the frequency division duplex (FDD) mode, while all base stations (BSs) use the same frequency spectrum for downlink transmission. Stateof-the-art literature proved that the system performance is mainly limited by the existing co-channel interference. Hence, mitigating these effects would improve the achievable system throughput beyond current benchmarks of cellular systems. Therefore, coordinated multi-point (CoMP) joint transmission (JT) is currently studied in Third Generation Partnership Project (3GPP). However, feedback demands due to massive channel state information (CSI) feedback cause a significant problem. By combining sub-clustering concepts for cooperative BSs with linear channel prediction methods in a thorough time-variant system-level analysis, we show that CSI feedback rates can be scaled down to less than $200 \mathrm{kBit} / \mathrm{s}$ per user.
\end{abstract}

\section{INTRODUCTION}

Various methods to combat co-channel interference (CCI) in cellular MIMO systems are summarized in [1], ranging from interference canceling receivers over multi-user diversity to closed-loop joint transmit beam-forming from multiple BSs. The joint transmission approach falls in the group of CoMP transmission, where multiple BSs transmit in a coordinated manner so that multiple data streams can be delivered simultaneously to different users in adjacent cells with reduced CCI. In the 3GPP standardization activities concerning Long Term Evolution - Advanced (LTE-A) [2], CoMP is considered as a tool to improve the coverage of high data rates and cell edge throughput. For CoMP MIMO, channel state information at the transmitter (CSIT) from multiple BSs and user equipments (UEs) is required. This can be achieved by measuring the channel in reverse direction and exploiting reciprocity of time division duplex (TDD) systems. In FDD systems, which are in the focus of this work, the channel is measured in the downlink direction at the UE side and then CSI is fed back via the uplink to the BSs.

Early concepts considered a huge centralized wireless distributed network (WDN), where all BS antenna are connected via a fast backhaul link to a central unit (CU) [3]-[5]. This setting corresponds to the MIMO broadcast channel (BC). Nonlinear signal pre-processing, known as dirty paper precoding [6], was shown to achieve the BC capacity [7]-[9]. In [10] authors relaxed the constraint of full data and CSI exchange for the case of static and arbitrarily varying interference. They focused on the case of two BSs, exchanging only partial information about their messages. By employing the Willems' conferencing protocol [11], authors proved that the single-user sum capacity can already be achieved without full information exchange. This study clearly motivates the development of CoMP schemes involving partial CSI and data sharing among cooperating BSs as e.g. discussed for coordinated scheduling and beamforming concepts [12]-[15] as well as interference alignment techniques [16]-[18].

For the use of JT CoMP in practice the overhead for pilots, feedback and backhaul has to be kept within reasonable limits. The most efficient way to reduce overhead is to limit the cooperation area. Therefore, we consider a small sub-cluster of BSs within a large cloud RAN (CRAN) area, where joint processing is only allowed between BSs belonging to the same CRAN entity. BSs outside the CRAN group are not coordinated and thus cause residual inter-cluster interference. Note, dynamic clustering reduces channel feedback as well as backhaul data exchange. Finally, we compare our system study with linear channel prediction in a quasi-static user regime. By reducing feedback over time, we show that CSI feedback rates can be reduced significantly at rather low costs in data throughput.

\section{TIME-VARIANT Downlink System Model}

We consider a cellular OFDM downlink where a central site is surrounded by multiple tiers of sites. We assume each site to be partitioned into three $120^{\circ}$ sectors, i.e. a set $\mathcal{M}$ consisting of $M=|\mathcal{M}|$ sectors in total. Each sector constitutes a cell, and frequency resources are fully reused in all $M$ cells. $\mathcal{M}_{c}$ represents the set of cells included in a given cluster and $M_{c}=\left|\mathcal{M}_{c}\right|$ denotes its size. At this stage, we assume disjoint clusters, i.e. a given BS cannot belong to more than one cluster operated at the same time/frequency resource. For OFDM systems, the overlap of multiple clusters can be achieved conveniently in the frequency domain.

Each cluster selects a set of active users $\mathcal{K}_{c}$ following a specific scheduling metric. In the $c$-th cluster, there are $M_{c}$ BSs, each one equipped with $N_{t}$ transmit antennas, while the $K_{c}=\left|\mathcal{K}_{c}\right|$ users are equipped each with $N_{r}$ receive antennas. The users inside the cluster are served by signals jointly emitted from $M_{c} N_{t}$ transmit antennas, where $M_{c} \cdot N_{t} \geq K_{c} \cdot N_{r}$. The $M_{c} N_{t} \times M_{c} N_{t}$ precoding matrix $\mathbf{B}_{c}=\left[\mathbf{B}_{c, 1} \cdots \mathbf{B}_{c, K_{c}}\right]$ contains the precoders $\mathbf{B}_{c, k}$ designed for each of the users. 
Note, each UE might receive multiple spatial layers at the same time, i.e. $\mathbf{B}_{c, k}$ is of dimension $M_{c} N_{t} \times\left|\mathcal{T}_{s, k}\right|$, where $\mathcal{T}_{s, k}$ denotes the set of spatial layers selected for instantaneous downlink service at user $k$. The maximum number of entries in this set is limited by the number of receive antennas, i.e. $\left|\mathcal{T}_{s, k}\right| \leq N_{r}$. The set $\mathcal{T}_{s}$ combines all selected spatial layers for spatial division multiple access (SDMA) service of the UEs in $\mathcal{K}_{c}$.

In order to describe the effect of outdated channel knowledge, we extend the general system model by a discrete time index $n$. We define $x_{c, t}(n)$ as the data symbol to be transmitted on the downlink and $\mathbf{H}_{c, k}(n)$ as the MIMO channel matrix at time instance $n .\left[\mathbf{B}_{c, k}\right]_{:, t}(n-\tau)$ denotes the precoding matrix used at time $n$ but based on a $\tau$-old estimate of the channel. Let us assume the precoder to follow a zero-forcing ( $\mathrm{ZF}$ ) constraint, which can be obtained using the Moore-Penrose pseudo inverse in case of MISO CSI feedback

$\left[\mathbf{B}_{c, k}\right]_{:, t}(n-\tau)=\mathbf{H}_{v i r t}^{H}(n-\tau)\left[\mathbf{H}_{v i r t}(n-\tau) \mathbf{H}_{v i r t}^{H}(n-\tau)\right]^{-1}$

The total transmit power is equally distributed among all active data streams, while we maintain a per antenna power constraint (PAPC) by using a simplified solution from [19]. The transmit power per antenna is chosen according to the row element in $\mathbf{B}_{c}$ with highest norm.

For further analysis, we assume the $c$-th cluster is surrounded by $M-M_{c}$ BSs evoking non-coordinated CCI. Thus, the received downlink signal $\mathbf{y}_{k, t}(n)$ for $t \in \mathcal{T}_{s, k} \subset \mathcal{T}_{s}$ at user $k$ in the cellular environment at the discrete time index $n$ is given by

$$
\begin{aligned}
& \mathbf{y}_{k, t}(n)=\mathbf{H}_{c, k}(n)\left[\mathbf{B}_{c, k}\right]_{:, t}(n-\tau) \sqrt{p_{c, t}} x_{c, t}(n) \\
& +\underbrace{\sum_{j \in \mathcal{T}_{s} \backslash\{t\}} \mathbf{H}_{c, k}(n)\left[\mathbf{B}_{c}\right]_{:, j}(n-\tau) \sqrt{p_{c, j}} x_{c, j}(n)}_{\boldsymbol{\vartheta}_{k, t}}+\mathbf{z}_{k}(n)
\end{aligned}
$$

$\left[\mathbf{B}_{c, k}\right]_{:, t}$ denotes the $t$-th column element in the global precoding matrix $\mathbf{B}_{c}$, where $\mathbf{B}_{c, k}$ correspond to the column elements designed for user $k$. The desired $t$-th data stream is distorted by the intra-cluster and inter-cluster interference plus noise aggregated in $\boldsymbol{\vartheta}_{k, t}$ and $\mathbf{z}_{k}$, respectively. $\mathbf{H}_{c, k}$ spans the $N_{r} \times M_{c} N_{t}$ channel matrix for user $k$ formed by the $c$-th cluster and $p_{c, t}$ is its power allocation valid for the $t$-th data stream. Thus, $\boldsymbol{\vartheta}_{k, t}$ denotes the interference generated within the cluster. ${ }^{1}$ The $N_{r} \times 1$ vector $\mathbf{n}$ denotes the additive white Gaussian noise (AWGN) samples with covariance $\mathbb{E}\left\{\mathbf{n n}^{H}\right\}=\mathbf{I} \sigma_{n}^{2}$. The noise power consists of the receiver noise figure and the thermal noise power.

The achievable signal-to-interference-and-noise ratio (SINR) 0 .

\footnotetext{
${ }^{1}$ Note, under ideal conditions and a ZF precoding constraint $\left|\mathbf{w}_{k, t}^{H} \boldsymbol{\vartheta}_{k, t}\right|=$
}

is estimated at each UE, according to

$$
\operatorname{SINR}_{k, t}(n)=\frac{\left|\mathbf{w}_{k, t}^{H}(n) \mathbf{H}_{c, k}(n)\left[\mathbf{B}_{c, k}\right]_{:, t}(n) \sqrt{p_{c, t}}\right|^{2}}{\left|\mathbf{w}_{k, t}^{H}(n)\left[\boldsymbol{\vartheta}_{k, t}(n)+\mathbf{z}_{k}(n)\right]\right|^{2}},
$$

with $\mathbf{w}_{k, t}$ being the combining weights at the $k$-th receiver and for data stream $t$.

The channel feedback is assumed as MISO CSI according to our work in [20], in particular focusing on a so-called multi-user eigenmode transmission (MET) strategy. The MET strategy is an extension of the block diagonalization (BD) concept, which was proposed in [21], [22] and uses a linear transmission strategy based on zero-forcing beamforming for maximizing the weighted sum rate. On a frame-by-frame basis, MET distributes up to $M_{c} N_{t}$ spatially multiplexed streams for one or multiple users. MET was initially proposed for multiuser MIMO (MU-MIMO) transmission [21] and was later utilized to cover the CoMP case [20]. According to the concept in [21], [23], the UEs are assumed to use linear receive filters $\boldsymbol{v}_{k}$ to transform their MIMO channel into an effective MISO channel. In essence, each user is assumed to multiply its channel matrix $\mathbf{H}_{c, k}=\mathbf{U}_{c, k} \boldsymbol{\Sigma}_{c, k} \mathbf{V}_{c, k}^{H}$ with a set $\mathcal{T}_{k}$ of Hermitian left dominant eigenvectors (4), i.e. the column vectors $\left[\mathbf{U}_{c, k}\right]_{:, t}$ included in $\mathbf{U}_{c, k}$ which correspond to the $\left|\mathcal{T}_{k}\right|$ strongest Eigenvalues in $\boldsymbol{\Sigma}_{c, k}$, i.e. $\boldsymbol{\Upsilon}_{k}=\left[\left[\mathbf{U}_{c, k}\right]_{:, 1} \ldots\left[\mathbf{U}_{c, k}\right]_{:,\left|\mathcal{T}_{k}\right|}\right]$.

$$
\begin{aligned}
{\left[\hat{\mathbf{H}}_{k}\right]_{t,:} } & =\left[\mathbf{U}_{c, k}\right]_{:, t}^{H} \mathbf{H}_{c, k} \\
& =\left[\mathbf{U}_{c, k}\right]_{:, t}^{H} \mathbf{U}_{c, k} \boldsymbol{\Sigma}_{c, k} \mathbf{V}_{c, k}^{H}=\left[\boldsymbol{\Sigma}_{c, k}\right]_{t, t}\left[\mathbf{V}_{c, k}\right]_{:, t}^{H}
\end{aligned}
$$

For a reduction in feedback rate, we assume each user is allowed to report its dominant eigenmode only, i.e. the first row element in $\left[\hat{\mathbf{H}}_{k}\right]_{t,:}$ :

In general, the scheme maximizes the signal power transfered from the collaborative BSs to the specific UE by using the dominant channel direction experienced by the receiver. The maximum received power would be achieved, if the precoder and receive filters are selected according to the right and left hand side matrices containing the corresponding eigenvectors $\left[\mathbf{V}_{c, k}\right]_{:, t}$ and $\left[\mathbf{U}_{c, k}\right]_{:, t}^{H}$, respectively. However, the $t$-th precoder significantly depends on the selected set of active users $\mathcal{K}_{c}$. In practice, the performance for the $k$-th user will be inferior. Thus, multiple UEs should be grouped preferably so that their effective channels $\hat{\mathbf{H}}_{k}$ show highest orthogonality. In general, appropriate user grouping keeps the costs in terms of a reduced received power after channel aware precoding as small as possible [24].

\section{Multi-Cell Simulations}

The radio channel traces are generated using the quasideterministic radio channel generator (QuaDRiGa), recently released by Fraunhofer Heinrich Hertz Institute [25]. Aiming for channel models being able to emulate precise time evolving for terrestrial cellular MIMO communications, QuaDRiGa 
TABLE I

SIMULATION ASSUMPTIONS.

\begin{tabular}{|c|c|}
\hline Par & Value \\
\hline $\begin{array}{l}\text { Drops } \\
\text { Channel evolution } \\
\text { Scenario } \\
\text { Propagation } \\
\text { Large-scale fading } \\
\text { Traffic model } \\
f_{c} \\
\text { Velocity, } f_{D}, T_{c} \\
\text { Frequency reuse } \\
\text { Signal bandwidth } \\
\text { Inter-site distance }\end{array}$ & $\begin{array}{r}\text { QuaDRiGa } \\
\text { Monte Carlo plus time evolution } \\
500 \\
500 \mathrm{~ms} \text { with } 1 \mathrm{~ms} \text { resolution } \\
\text { Urban-macro } \\
\text { NLOS } \\
\text { Geo-correlated parameters maps } \\
\text { Full buffer } \\
2.6 \mathrm{GHz} \\
3 \mathrm{~km} / \mathrm{h}, 7.5 \mathrm{~Hz}, 133 \mathrm{~ms} \\
1 \\
18 \mathrm{MHz}, 100 \mathrm{PRBs} \\
500 \mathrm{~m}\end{array}$ \\
\hline $\begin{array}{l}\text { Beamforming } \\
\text { Clustering } \\
\text { User grouping }\end{array}$ & $\begin{array}{r}19 \text { having } 3 \text { sectors each } \\
4 ; 4 \lambda \\
46 \mathrm{dBm} \\
\text { Azimuth: FWHM of } 58^{\circ} \\
\text { Elevation: FWHM of } 6.2^{\circ} \\
10^{\circ} \text { electrical downtilt } \\
32 \mathrm{~m} \\
\text { Cluster-wide MET, ZF constraint } \\
\text { Network-centric } \\
3 \text { user per sector, round-robin }\end{array}$ \\
\hline $\begin{array}{l}N_{r} ; \text { spacing } \\
\text { UE height } \\
\text { Link-2-System } \\
\text { CSI delay } \\
\text { Channel prediction }\end{array}$ & $\begin{array}{l}2 ; \lambda / 2 \\
2 \mathrm{~m} \\
\text { Shannon, SINRs }\lfloor-10,40\rceil \mathrm{dB} \\
\{0,5,10,20,40,80,120\} \mathrm{ms} \\
\text { RLS with } d=1 \mathrm{~ms} \text { and } \alpha \text { set } \\
\text { to the considered feedback delay }\end{array}$ \\
\hline
\end{tabular}

follows the modeling principles of geometry based stochastic channel models like SCME, WINNER+ and the COST 2100 channel model. Interested readers may refer to [26] for the main features of the generator and channel models. For this study, large-scale channel parameters are chosen according to latest parametrization guidelines from WINNER+. A detailed list of the properties of the multi-cell channel and corresponding system-level simulation parameters are given in Table I. The achievable data rates are determined from the SINRs after equalization according to expression (3). These post-equalization SINRs are mapped into data rates using a Shannon's formula. Note, input SINRs are mapped to a valid range $\lfloor-10,40\rceil \mathrm{dB}$.

\section{Sub-Clustering and Greedy Sum Rate MAXIMIZATION}

According to our work in [27], we allow UEs belonging to a certain CRAN cluster $\mathcal{M}_{c}$ to select their desired sub-set of BSs for cooperative downlink transmission. We extend a static and network-centric clustering towards a user-driven selection of sub-clusters $\mathcal{M}_{c, k} \subset \mathcal{M}_{c}$. Each user can choose its desired subset e.g. based on the given received power. This results into an overlapping definition of sub-clusters within a static, network-centric cluster $\mathcal{M}_{c}$. As a result, the overall feedback matrix is rather sparse, since non-reported CSI values are filled up with zero entries. This sub-clustering approach reduces channel estimation complexity at the UE side, i.e. only the set of strongest BSs needs to estimated and simply saves feedback overhead. Note, weaker signals, which typically yield higher channel estimation errors, are ignored within this approach and thus the overall CSIT representation is more robust.

In order to reduce the computational complexity at the BS side, we combine a greedy user search [21] with a rate approximation criteria described in [28]. Fuchs et al. suggest a method replacing the singular value decomposition (SVD) operations by an approximation using subsequent orthogonal projections into the separate users' null-spaces. Therefore, let us define a matrix $\tilde{\boldsymbol{\Psi}}_{k}$ which projects the channel matrix $\hat{\mathbf{H}}_{k}$ of user $k$ into the null-space of $\tilde{\mathbf{H}}_{k}$. $\tilde{\mathbf{H}}_{k}$ contains the others users' channel matrices and choosing any projection into its null-space $\tilde{\mathbf{V}}_{k}^{0}$ for downlink service of user $k$ fulfills the ZF condition. The projector of user $k$ is based on the CSI feedback of each user,

$$
\boldsymbol{\Psi}_{k}=\mathbf{I}-\underbrace{\hat{\mathbf{H}}_{k}^{H}\left(\hat{\mathbf{H}}_{k} \hat{\mathbf{H}}_{k}^{H}\right) \hat{\mathbf{H}}_{k}}_{\begin{array}{c}
=\mathbf{V}_{c, k} \mathbf{V}_{c, k}^{H} \\
\text { in case of MET }
\end{array}}
$$

The projection matrix $\tilde{\Psi}_{k}$, which combines all users' nullspace except the $k$-th UE, can be approximated by repeatedly applying

$$
\tilde{\mathbf{\Psi}}_{k}=\left[\boldsymbol{\Psi}_{1} \cdot \ldots \cdot \boldsymbol{\Psi}_{k-1} \boldsymbol{\Psi}_{k+1} \cdot \ldots \cdot \boldsymbol{\Psi}_{K_{c}}\right]^{n},
$$

with $n \rightarrow \infty$ as the projection order. Authors in [28] state, that for small projection order, i.e. $n \in\{1, \ldots, 4\}$ the user grouping performance is already sufficient. As stated in [29], we use

$$
R_{k}=\log _{2}\left(1+\frac{p_{c, k}}{\sigma_{n}^{2}}\left\|\hat{\mathbf{H}}_{k} \tilde{\mathbf{\Psi}}_{k}\right\|_{F}^{2}\right)
$$

as an estimate to assess the link rate for the $k$-th UE. ${ }^{2}$ It may be considered as a lower bound for ZF capacity which may be obtained by using BD. The scheduling entity collects the projectors of already selected UEs and determines the tentative next user by evaluating (7) for all residual users or receive spaces. The UE with maximum link rate, i.e.

$$
k: \underset{k \in \mathcal{K}_{c} \backslash \mathcal{T}_{s}}{\arg \max }\left\|\hat{\mathbf{H}}_{k} \tilde{\mathbf{\Psi}}_{k}\right\|_{F}^{2}
$$

is added to the group of already scheduled users' receive spaces $\mathcal{T}_{s}$. We found that this greedy rate approximation (GRA) metric adaptively loads up to $0.75 N_{t}$ data streams per sector in the cluster. As an extension, we may change the metric from maximum rate to a metric which ensures some degree of fairness. By employing the score-based metric, we may ensure that each UE is assigned an equal amount of resources within a given time window.

Fig. 1 summarizes these findings for GRA in terms of the median sum-rate per sector as a function of the sub-cluster size $M_{c, k}$. The sum-rate per sector is increasing logarithmic with increasing cluster size, while we observe an additional increase

\footnotetext{
${ }^{2}$ Note, the term does not incorporate inter-cluster interference in the user selection process. This may lead to non-optimal selection of UEs with respect to the resulting sum-rate.
} 


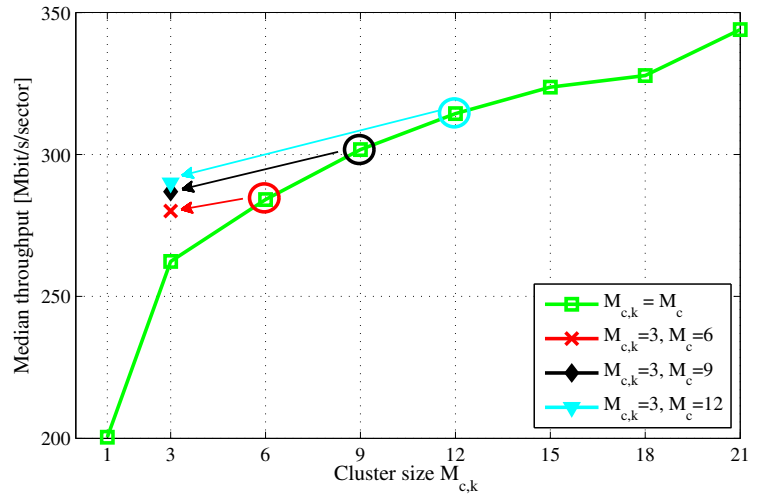

Fig. 1. Median achievable system rate as a function of sub-cluster size $M_{c, k}=M_{c}$, green line. The results for a fixed sub-cluster size of $M_{c, k}=3$ and an increasing cluster $M_{c}=\{6,9,12\}$ are given as red cross, black diamond and cyan triangle, respectively.

for $M_{c, k}=21$ caused by the topology of the cluster definition from [27]. On the other hand, the feedback data rate will scale linearly, compare section VI. Furthermore, we consider the case of $M_{c, k}=3$ where the cluster size continuously grows from $M_{c}=\{3,6,9,12\}$, i.e. each user can select its desired sub-cluster out of a growing, static cluster. It is obvious, that with $M_{c}=12$ there is significantly higher chance of finding the globally strongest three sectors compared to $M_{c}=3$. For a specific pair of cluster size $x$ and sub-cluster size $y$ we will use the following short notation $\langle x, y\rangle$. From $\langle 3,3\rangle$ to $\langle 6,3\rangle$ a gain of $7 \%$ is shown due to selection diversity of the subcluster $\mathcal{M}_{c, k}$. This is confirmed by a further increase of $3 \%$ for $\langle 9,3\rangle$ and $1 \%$ for $\langle 12,3\rangle$.

\section{Linear AdAptive ChanNEl PREdiction}

Adaptive algorithms, e.g. the well-known normalized leastmean-squares (NLMS) and recursive least squares (RLS) filters [30], can be designed to converge towards the optimal solution of Wiener W $_{M \rightarrow \infty}$. However, they need to be trained to adapt to the current channel statistics while a so-called forgetting factor $\lambda$ steers the impact of $\tau$-old channel estimates. An appropriate selection of $\lambda$ is mandatory in order to optimize the trade-off between excess mean square error (EMSE) and convergence time. For the RLS filter, we chose the QR-decomposition (QRD) based implementation since it has superior numerical properties in finite precision. Note, that there exists fast RLS algorithms with only $O(p)$ operations per iteration [30, Ch. 37-43], as opposed to $O\left(p^{2}\right)$ in case of the classical implementation, which may be applied to this problem. Since those variants are more difficult to implement than QRD-RLS, and computational complexity is not an issue in our work, we do not consider them further.

According to our initial studies in [27] for linear channel prediction based on adaptive RLS method we configure the parameters of the filter to ensure a small EMSE after a system's settling of $400 \mathrm{~ms}$ with a sampling resolution of $1 \mathrm{~ms}$. In particular that means, the forgetting factor and the regularization parameter are chosen to $\lambda=0.99$ and $\epsilon=0.01$,

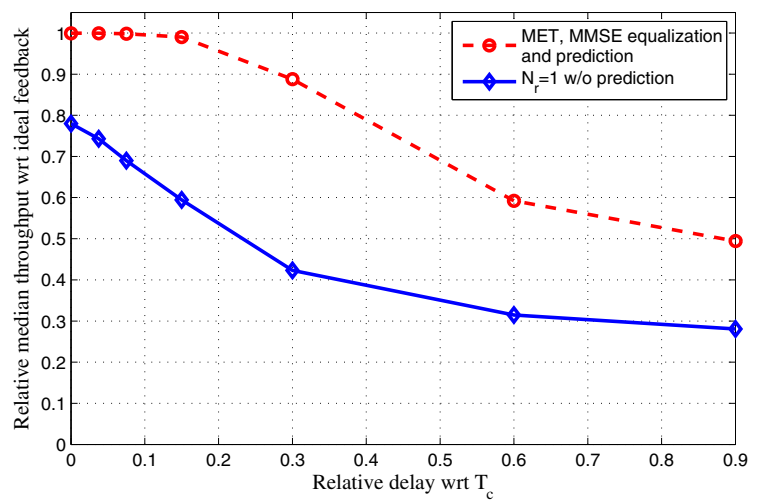

Fig. 2. Simulation results for $M_{c}=1$, i.e. single-cell ZF precoding, taking precise channel evolution into account and specific channel feedback interval. Where the coherence time is set to the inverse of the maximal Doppler shift, i.e. $T c=1 / f_{D}$.

respectively. The prediction horizon is configured to cover the selected feedback delay $\tau$.

Figure 2 depicts the loss due to channel aging, while the $\mathrm{x}$-axis is scaled in intervals of the coherence time $T_{c}$. The $\mathrm{y}$ axis is normalized to the maximum achievable data rate for $\tau=0 \mathrm{~ms}$ feedback delay, i.e. the scheme which performs best in the current study under ideal feedback conditions. In general, we assume CSI feedback based on the MET metric, while the UEs use minimum mean square error (MMSE) combining weights based on ideal channel knowledge for receive processing. For comparison, we also include the configuration of single-antenna receivers, i.e. $N_{r}=1$ without channel prediction capabilities. At this point, we can conclude with roughly $112 \%$ gain in median system throughput for advanced receivers over baseline assumptions at $0.3 T_{c}$ for the tested range of cluster size $M_{c}$.

\section{REQUiREd FEEDBACK RATE}

From Fig. 2 we conclude, a feedback interval of $20 \mathrm{~ms}$ results in a loss in data rate of approximately $1 \%$. With $40 \mathrm{~ms}$ delay the overall loss in sum-rate increases to almost $11 \%$. Note, with increasing cluster size $M_{c, k}$ the influence from channel aging is getting more prominent since errors superimpose. Let us assume a sub-cluster size of $M_{c, k}=3$. Thus, $\mathbf{H}_{c, k}$ consists of $\mathcal{T}_{k} N_{t} M_{c, k}=12$ sub-channels. ${ }^{3}$ Considering the coherence bandwidth of urban-macro channels, it should be sufficient to report those sub-channels once per resource block (RB), i.e. the feedback rate $R_{f b, k}$ attributes to

$$
\begin{aligned}
R_{f b, k} & =12 \cdot \mathcal{T}_{k} N_{t} M_{c, k} \cdot 100 /\left(20 \cdot 10^{-3}\right) \mathrm{bit} / \mathrm{s} \\
& =0.720 \mathrm{Mbit} / \mathrm{s},
\end{aligned}
$$

assuming feedback for all 100 physical resource blocks (PRBs) in $18 \mathrm{MHz}$ bandwidth, 12 Bit quantization in amplitude and phase information per sub-channel but with a time resolution of $20 \mathrm{~ms}$. We can further reduce the feedback rate by allowing a higher loss in data rate due to feedback outdating or more convenient by constraining the feedback to a sub-band only.

\footnotetext{
${ }^{3}$ Assuming $\mathcal{T}_{k}=1$, i.e. feedback for a single spatial layer per UE.
} 
E.g. allowing each UE to report CSI for its best $25 \%$ of PRBs, we simply scale down the feedback requirement to $180 \mathrm{kBit} / \mathrm{s}$ at moderate cost in sum-rate reduction.

\section{CONCLUSION}

In this work, we demonstrated the data rate scaling behavior of clustered CoMP transmission. Since network backhaul topology will significantly influence the capability of setting up clusters for CoMP transmission, we assume network-centric clustering as e.g. defined by a CRAN architecture. In order to save precious uplink resources for CSI feedback, we suggest to enable user-driven sub-clustering. We demonstrated that MIMO processing at both ends of the link can improve system as well as user performance in presence of outdated CSI feedback. Finally, we conclude with the observation of a rather small loss in data rate at a feedback delay of $15 \%$ of the coherence time of the channel. In essence, by using simple 1-dimensional, adaptive channel prediction we can allow CSI feedback once per $20 \mathrm{~ms}$ in the quasi-static user regime of $3 \mathrm{~km} / \mathrm{h}$ velocity. Finally assuming, that each UE does not provide CSI feedback over the whole range of PRBs, channel feedback can be reduced to less than $200 \mathrm{kBit} / \mathrm{s}$.

\section{ACKNOWLEDGEMENTS}

Part of this work has been performed in the framework of the FP7 project ICT-317669 METIS, which is partly funded by the European Union. The authors would like to acknowledge the contributions of their colleagues in METIS, although the views expressed are those of the authors and do not necessarily represent the project.

\section{REFERENCES}

[1] J. Andrews, W. Choi, and R. Heath, "Overcoming interference in spatial multiplexing MIMO cellular networks," IEEE Wireless Communications, vol. 14, no. 6, pp. 95-104, Dec. 2007.

[2] TR 36.814 V1.0.0, "Evolved universal terrestical radio access (EUTRA); further advancements for (E-UTRA) physical layer aspects," Feb. 2009

[3] P. Baier, M. Meurer, T. Weber, and H. Troger, "Joint transmission (JT), an alternative rationale for the downlink of time division CDMA using multi-element transmit antennas," Spread Spectrum Techniques and Applications, 2000 IEEE Sixth International Symposium on, vol. 1, pp. 1-5 vol.1, 2000

[4] S. Shamai and B. Zaidel, "Enhancing the cellular downlink capacity via co-processing at the transmitting end," IEEE Vehicular Technology Conference, VTC 2001 Spring, vol. 3, pp. 1745 -1749 vol.3, 2001.

[5] A. Goldsmith, S. Jafar, N. Jindal, and S. Vishwanath, "Capacity limits of MIMO channels," IEEE Journal on Selected Areas in Communication, vol. 21, no. 5, pp. 684-702, Jun. 2003.

[6] M. Costa, "Writing on dirty paper (corresp.)," IEEE Transactions on Information Theory, vol. 29, no. 3, pp. 439-441, 1983.

[7] G. Caire and S. S. (Shitz), "On the achievable throughput of a multiantenna Gaussian broadcast channel," IEEE Transactions on Information Theory, vol. 49, no. 7, pp. 802-811, Jun. 2003.

[8] P. Viswanath and D. Tse, "Sum capacity of the vector Gaussian broadcast channel and uplink-downlink duality," IEEE Transactions on Information Theory, vol. 49, no. 8, pp. 1912-1921, 2003.

[9] N. Jindal, S. Vishwanath, and A. Goldsmith, "On the duality of Gaussian multiple-access and broadcast channels," IEEE Transactions on Information Theory, vol. 50, no. 5, pp. 768-783, 2004.

[10] M. Wiese, H. Boche, I. Bjelaković, and V. Jungnickel, "The compound multiple access channel with partially cooperating encoders," IEEE Transactions on Information Theory, vol. 57, no. 5, pp. $3045-3066$, May 2011.
[11] F. Willems, "The discrete memoryless multiple access channel with partially cooperating encoders," IEEE Transactions on Information Theory, vol. 29, no. 3, pp. 441 - 445, May 1983.

[12] D. Gesbert, S. Kiani, A. Gjendemsj, and G. ien, "Adaptation, coordination, and distributed resource allocation in interference-limited wireless networks," Proceedings of the IEEE, vol. 95, no. 12, pp. $2393-2409$, dec. 2007.

[13] C.-B. Chae, D. Mazzarese, T. Inoue, and R. Heath, "Coordinated beamforming for the multiuser MIMO broadcast channel with limited feedforward," IEEE Transactions on Signal Processing, vol. 56, no. 12, pp. $6044-6056$, Dec. 2008.

[14] H. Huang, M. Trivellato, A. Hottinen, M. Shafi, P. Smith, and R. Valenzuela, "Increasing downlink cellular throughput with limited network MIMO coordination," IEEE Transactions on Wireless Communication, vol. 8, no. 6, pp. 2983 -2989, Jun. 2009.

[15] J. Giese and M. Amin, "Performance upper bounds for coordinated beam selection in LTE-Advanced," in International ITG Workshop on Smart Antennas (WSA), Feb. 2010, pp. 280 -285.

[16] J. Schreck and G. Wunder, "Interference alignment over limited dimensions for cellular networks: Feasibility and algorithms," in International ITG Workshop on Smart Antennas (WSA), Mar. 2012, pp. 352 -358.

[17] D. Aziz, F. Boccardi, and A. Weber, "System-level performance study of interference alignment in cellular systems with base-station coordination," 23rd IEEE Intern. Symp. on Personal, Indoor and Mobile Radio Communications (PIMRC 2012), Sep. 2012.

[18] O. E. Ayach, S. W. Peters, and R. W. H. Jr., "The practical challenges of interference alignment," CoRR, vol. abs/1206.4755, 2012.

[19] H. Zhang and H. Dai, "Cochannel interference mitigation and cooperative processing in downlink multicell multiuser MIMO networks," Eurasip Journal on Wireless Communications and Networking, vol. 2004, no. 2, pp. 222-235, 2004.

[20] L. Thiele, T. Wirth, T. Haustein, V. Jungnickel, E. Schulz, and W. Zirwas, "A unified feedback scheme for distributed interference management in cellular systems: Benefits and challenges for real-time implementation," 17th Euopean Signal Processing Conference (EUSIPCO2009), Aug. 2009, invited.

[21] F. Boccardi and H. Huang, "A near-optimum technique using linear precoding for the MIMO broadcast channel," IEEE International Conference on Acoustics, Speech and Signal Processing (ICASSP), vol. 3, pp. III-17-III-20, Apr. 2007.

[22] M. Trivellato, F. Boccardi, and H. Huang, "On transceiver design and channel quantization for downlink multiuser MIMO systems with limited feedback," IEEE Journal on Selected Areas in Communications, vol. 26, no. 8, pp. 1494-1504, Oct. 2008.

[23] M. Noda, M. Muraguchi, T. G. Khanh, K. Sakaguchi, and K. Araki, "Eigenmode tomlinson-harashima precoding for multi-antenna multiuser mimo broadcast channel," in 6th International Conference on Information, Communications Signal Processing, 10-13 2007, pp. 1 -5.

[24] L. Thiele, M. Schellmann, T. Wirth, V. Jungnickel, F. Boccardi, and H. Huang, "DFT-based vs. cooperative MET-based MU-MIMO in the downlink of cellular OFDM systems," International ITG Workshop on Smart Antennas (WSA 2009), Feb. 2009.

[25] Fraunhofer-Gesellschaft, "Quasi-Deterministic Radio Channel Generator (QuaDRiGa)," Oct. 2012. [Online]. Available: http://quadriga-channelmodel.de/

[26] K. Börner, J. Dommel, S. Jaeckel, and L. Thiele, "On the requirements for quasi-deterministic radio channel models for heterogeneous networks," in International Symposium on Signals, Systems and Electronics (ISSSE'12), Oct. 2012, pp. 1-5.

[27] L. Thiele, M. Kurras, K. Borner, and T. Haustein, "User-aided subclustering for CoMP transmission: Feedback overhead vs. data rate trade-off," 46th Asilomar Conference on Signals, Systems and Computers, Nov. 2012, accepted.

[28] M. Fuchs, G. D. Galdo, and M. Haardt, "Low complexity spatial scheduling ProSched for MIMO systems with multiple base stations and a central controller," in International ITG/IEEE Workshop on Smart Antennas (WSA), (Guenzburg, Germany), Mar. 2006.

[29] M. Fuchs, G. Del Galdo, and M. Haardt, "Low-complexity spacetime-frequency scheduling for MIMO systems with SDMA," IEEE Transactions on Vehicular Technology, vol. 56, no. 5, pp. 2775 -2784, Sep. 2007.

[30] A. H. Sayed, Adaptive Filters. Wiley-Interscience, 2008 